\title{
Policies vs Practice of Medical Error Disclosure at a Teaching Hospital in Saudi Arabia
}

This article was published in the following Dove Press journal:

Risk Management and Healthcare Policy

\author{
Reem Al Madani' \\ Saja A Al-Rayes $\mathbb{D}^{2}$ \\ Arwa Alumran $\mathbb{D}^{2}$
}

'Risk Management Unit, Directorate of Quality and Safety, King Fahad Hospital of the University, Imam Abdulrahman Bin Faisal University, Dammam, Saudi Arabia; ${ }^{2}$ Health Information Management and Technology Department, College of Public Health, Imam Abdulrahman Bin Faisal University, Dammam, Saudi Arabia

Correspondence: Reem Al Madani Risk Management Unit, Directorate of Quality and Safety, King Fahad Hospital of the University, Imam Abdulrahman Bin Faisal University, PO Box 38954,

Dahahran 31932, Saudi Arabia

$\mathrm{Tel}+96638966666$

Email raalmadani@iau.edu.sa
Background: Medical errors are unavoidable in health care institutions. Errors can occur due to multiple reasons, yet communication between health care providers has proven to be the highest. However, policies and programs of medical error disclosure were established to ensure that patients and their family members get the necessary closure. Hence, it is vital to recognize physicians' awareness of policies and programs related to disclosure practice.

Objective: The objective of this study was to examine factors impacting the awareness of hospital policies and programs and their impact on the actual disclosure of medical errors.

Methods: This was a quantitative cross-sectional study, using a self-administered survey given to 206 physicians from numerous departments at King Fahd Hospital of the University. Results: The majority of participants were not aware of policies and programs related to disclosure, nor had they disclosed a medical error to patients. There was no statistical significance between policies and programs and the practice of disclosure, yet position level and gender were statistically significantly related to awareness.

Conclusion: Physicians of King Fahd Hospital of the University demonstrated a low awareness rate of the hospital policies and programs regarding disclosure practice. Advanced efforts of hospital leaders need to be made to communicate, train and educate providers about their policies to promote disclosure practice.

Keywords: medical errors, disclosure, policies, procedure

\section{Introduction}

Health care organizations internationally are establishing policies and programs to prevent medical errors, yet errors are still unavoidable in health care institutions worldwide. ${ }^{1}$ Error was defined by the Institute of Medicine as the "failure of a planned action to be completed as intended," while mistake was defined as "an unanticipated negative consequence of medical intervention". 2

A vital ethical role of physicians is to respect patients' autonomy and disclose medical errors. ${ }^{1}$ According to the disclosure of medical error policies, errors must be revealed to patients and their families; however, policymaking is extremely difficult in health care due to health care organizations' obligation toward patient-centered practice; adding patients' needs is a major part of creating health policy objectives. ${ }^{3}$

During the mid-1980s, a full-disclosure policy was implemented in the US, following major harm cases; several health care organizations followed the same lead. ${ }^{3}$ Furthermore, the National Patient Safety Foundation announced a report stating,

When a health care injury occurs, the patient and the family or representative are entailed to a prompt explanation of how the injury occurred and long-term effects. ${ }^{2}$ 
During the mid-1990s, an Australian health care study announced that medical error is a major quality problem and found that $16 \%$ of patients admitted were associated with adverse events that were preventable, and the majority of patients preferred an honest acknowledgement of the error. $^{4}$

Efforts to decrease medical errors and improve the quality of care are the main focus of every health care organization. ${ }^{6}$ Moreover, disclosing medical errors to patients and families can improve community confidence toward health providers and their institutions. ${ }^{2}$ Hospitals are required by accreditation standards to inform patients and their families about medical errors by establishing policies and second victim programs of disclosure to ensure that patients and their family members get the necessary closure. ${ }^{1}$ Although policies, programs and guidelines were applied to define, measure and promote patient safety, as confirmed by Gallagher et al, ${ }^{1}$ there is no evidence worldwide that they actually increase disclosure practice. Therefore, there is a need to study this association to promote disclosure practice within health care sectors.

The aim of this study was to examine the factors that impact the awareness of disclosure policies and second victim programs, as well as the impact of policies and programs on the disclosure of medical errors at King Fahd Hospital of the University. The hospital established two policies and one program to support disclosure practice: first, the "Disclosure of Unanticipated Patient's Outcome" policy, which was created in early 2016 and defined the process of revealing information to patients and family regarding a medical error. ${ }^{5}$ Second, the "Just Culture" policy was also created in early 2016 and refers to the organization's recognition that individuals should not be held responsible for system and process failure; the policy also recognizes that competent physicians can make mistakes. On the other hand, the "Second Victim Program" that was created in 2019 refers to an activity designed to take action and support health care providers as soon as medical errors occur.

\section{Methods}

This study was a quantitative cross-sectional design. The participants were 206 physicians from numerous position levels and specialties recruited randomly from King Fahd Hospital of the University. The inclusion criteria included physicians at the resident level and above, employed at King
Fahd Hospital of the University, while the exclusion criteria were physicians below resident level and rotating physicians.

King Fahd Hospital of the University is a tertiary teaching hospital located in Al Khobar, Eastern Province, Saudi Arabia. The 634-bed-capacity hospital is accredited by the Joint Commission International and Central Board for Accreditation of Healthcare Institutions. The hospital has various departments, and the study included physicians from all hospital departments, nine specialties under surgical departments and eight specialties under medical departments. The study setting was purposely selected because it is a tertiary hospital staffed with many physicians, and the interaction between physicians and patients was bound to be very much elevated.

Participants in the study were physicians from numerous positions, including consultant physicians, specialists, fellows, and resident physicians, in addition to general practitioners. King Fahd Hospital has a total of 441 physicians, and using the sample size formula indicated by Daniel ${ }^{7}$ Sample size was calculated through the sample size formula: $\mathrm{n}=\mathrm{N} * \mathrm{X} /(\mathrm{X}+\mathrm{N}-1]$, where $\mathrm{N}$ is the population size which is $441, X=Z \alpha / 2^{2} * p^{*}(1-p) / M^{2} E^{2}$. The confidence interval is set to $95 \%$, Margin of error (MOE) " $\alpha$ " is 0.05 , and $p$ is the sample proportion, ie, 0.5 . Finally, $Z \alpha / 2$ is the critical value of the Normal distribution, which is set to 1.96. Therefore, the formula is $n=441 *\left[1.96^{2} * 0.5 *(1-0.5) / 0.05^{2}\right]$ showed that the appropriate sample size for the target population was 206 physicians.

Ethical approval was obtained from the Institutional Review Board, Office of Vice President for Research and Higher Studies at Imam Abdulrahman bin Faisal University with reference number IRB-PCG 2019-03-388.

The data collection instrument was developed for the aim of this study and was self-administered to 206 physicians to gather the needed information. The questionnaire was administered in morning report and office working hours. The survey included seven dominos, with three demographic variable questions about gender, specialty and position level; three questions about policies and programs, which served as independent variables; and one question about disclosure, which served as the dependent variable.

The Statistical Package for Social Sciences (SPSS) version $26^{8}$ was used to analyze the results. Univariate analysis was conducted to present the summary, and bivariate analysis was conducted to present the significant associations. Chisquare was used to measure the results because the outcome variable was binary. A $P$-value of less than 0.05 was considered significant in all statistical analyses in this study. 
To make sure that this study did not suffer from tawdriness, ${ }^{9}$ the questionnaire's face and content validity were assessed, therefore, 10 experts were interviewed about the survey validity; four of the experts were faculty members at Imam Abdulrahman Bin Faisal and six were physicians from the study setting (who were excluded from the final sample). This step was important to make sure the research did not suffer from poor quality.

\section{Results}

The self-administered survey was distributed to 300 physicians at King Fahd Hospital of the University, and only 206 physicians completed the survey (response rate 69\%). As shown in Table 1, the majority of the study sample were males $(71 \%)$, and most of the participants were consultants $(39 \%)$. Half of the participants were from medical departments (52\%).

Regarding participants' awareness about different policies and programs related to disclosure, the majority of physicians were aware of the "Disclosure of Unanticipated Patient's Outcome" policy (30\%), followed by the "Second Victim Program" (24\%) and "Just Culture" (23\%). Overall awareness was measured and showed mean score of 0.8 and median score of 0 , as shown in Table 1.

The associations between the participants' demographic characteristics and their awareness about different policies and programs in the study setting were measured.

Table I Demographic Characteristics of the Study Participants

\begin{tabular}{|l|l|}
\hline Demographic Variables & $\mathbf{n = 2 0 4}$ (\%) \\
\hline Position Level & \\
Consultants & $80(39.3)$ \\
Specialist & $38(18.9)$ \\
Fellows & $7(3.4)$ \\
Residents & $62(30.1)$ \\
GPs & $17(8.3)$ \\
\hline Gender & \\
Female & $60(29.3)$ \\
Male & $143(70.7)$ \\
Missing & $1(1)$ \\
\hline Departments & \\
Medical & $107(51.9)$ \\
Surgical & $97(48.1)$ \\
\hline Awareness & \\
Policy I & $62(30)$ \\
Policy 2 & $48(23.5)$ \\
Policy 3 & $49(24)$ \\
\hline
\end{tabular}

The results indicated that consultants were more aware (54\%) than staff in other positions, while $39 \%$ of residents were not aware of any policies. These results demonstrated statistically significant associations $\left(\mathrm{x}^{2}=23.452, P=0.000\right)$, as shown in Table 2 .

Moreover, male physicians were more aware of policies provided by the hospital ( $80 \%)$ compared to females. This difference was statistically significant $\left(\mathrm{x}^{2}=5.857\right.$, $P=0.016)$ (Table 2).

However, there appeared to be no significant difference between the departments (surgical and medical) regarding their awareness of the hospital disclosure policies $\left(\mathrm{x}^{2}=\right.$ $0.545, P=0.460$ ), as shown in Table 2 .

As shown in Table 2, overall, the physicians' awareness of disclosure policies provided by the hospital and its association with the actual disclosure practice appeared to be not significant $\left(\mathrm{x}^{2}=0.205, P=0.651\right)$. This indicates that awareness of policies and programs does not influence actual disclosure behavior.

Furthermore, Table 3 shows the relationship between each disclosure policy and the number of disclosure practices. There was no association between awareness about the abovementioned policies and the actual behavior of disclosing medical errors to patients.

Table 2 Factors Associated with Policies and Program Awareness

\begin{tabular}{|c|c|c|c|}
\hline Variable & $\begin{array}{l}\text { Not Aware } \\
\text { of Any } \\
\text { Policy } \\
n=124 \text { (\%) }\end{array}$ & $\begin{array}{l}\text { Aware of at } \\
\text { Least I } \\
\text { Policy } n=82 \\
\text { (\%) }\end{array}$ & Chi-Square Test \\
\hline $\begin{array}{l}\text { Position Level } \\
\text { Consultants } \\
\text { Specialist } \\
\text { Fellows } \\
\text { Residents } \\
\text { GPs }\end{array}$ & $\begin{array}{l}37(29.8) \\
19(15.3) \\
7(5.6) \\
48(38.7) \\
13(10.5)\end{array}$ & $\begin{array}{l}44(53.7) \\
20(24.2) \\
0(0) \\
14(17.1) \\
4(4.9)\end{array}$ & $\begin{array}{l}x^{2}=23.452 \\
P<0.0001\end{array}$ \\
\hline $\begin{array}{l}\text { Gender } \\
\text { Female } \\
\text { Male } \\
\text { Missing }\end{array}$ & $\begin{array}{l}44(35.5) \\
80(64.5) \\
0(0)\end{array}$ & $\begin{array}{l}16(19.8) \\
65(80.2) \\
I(1)\end{array}$ & $\begin{array}{l}x^{2}=5.857, P=0.016 \\
\text { Fisher exact test, } \\
P=0.018\end{array}$ \\
\hline $\begin{array}{l}\text { Departments } \\
\text { Medical } \\
\text { Surgical }\end{array}$ & $\begin{array}{l}67(54) \\
57(46)\end{array}$ & $\begin{array}{l}40(48.8) \\
42(51.2)\end{array}$ & $\begin{array}{l}x^{2}=0.545, P=0.460 \\
\text { Fisher exact test, } \\
P=0.479\end{array}$ \\
\hline $\begin{array}{l}\text { Disclosure } \\
\text { Disclosed } \\
\text { Never } \\
\text { Disclosed }\end{array}$ & $\begin{array}{l}49(39.5) \\
75(60.5)\end{array}$ & $\begin{array}{l}35(42.7) \\
47(57.3)\end{array}$ & $\begin{array}{l}x^{2}=0.205, P=0.651 \\
\text { Fisher exact test, } \\
P=0.666\end{array}$ \\
\hline
\end{tabular}


Table 3 The Influence of Awareness of Disclosure Policies on the Actual Behavior or Disclosure of Medical Errors to Patients

\begin{tabular}{|c|c|c|c|}
\hline \multirow[t]{2}{*}{ Variable } & \multicolumn{2}{|c|}{$\begin{array}{l}\text { Have You Ever Disclosed } \\
\text { a Medical Error to } \\
\text { a Patient? }\end{array}$} & \multirow[t]{2}{*}{ Test Statistic } \\
\hline & $\begin{array}{l}\text { Yes } \\
n=84 \text { (\%) }\end{array}$ & $\begin{array}{l}\text { No } \\
n=|2|(\%)\end{array}$ & \\
\hline \multicolumn{4}{|l|}{ Disclosure } \\
\hline & & & \\
\hline Yes & $25(40.3)$ & $37(59.7)$ & $x^{2}=0.001, P=0.981$ \\
\hline No & $57(40.2)$ & $85(59.8)$ & Fisher exact test, $P=1.000$ \\
\hline \multicolumn{4}{|l|}{ Just Culture } \\
\hline \multicolumn{4}{|l|}{ Policy } \\
\hline Yes & $14(29.2)$ & $34(70.8)$ & $x^{2}=3.176, P=0.075$ \\
\hline No & $68(43.5)$ & $88(56.4)$ & Fisher exact test, $P=0.092$ \\
\hline \multicolumn{4}{|l|}{ Second } \\
\hline \multicolumn{4}{|l|}{ Victim Policy } \\
\hline Yes & $18(36.7)$ & $31(63.3)$ & $x^{2}=0.305, P=0.581$ \\
\hline No & $63(4 I .1)$ & $90(58.8)$ & Fisher exact test, $P=0.619$ \\
\hline
\end{tabular}

Finally, detailed associations of factors influencing awareness of different policies and programs directed at the disclosure of medical errors to patients by the hospital administration are shown in Table 4.

\section{Discussion}

Disclosure policies and second victim programs intend to guarantee the implementation of organizational roles for good disclosure practice. ${ }^{1}$ In this study, we explored the association between the demographic factors "position level, gender and specialty" and awareness of policies and the "Second Victim Program" at King Fahd Hospital of the University. We found that consultants were more aware than staff members at other position levels, which indicated a significant association between position level and awareness of policies and programs. Kaldjian's ${ }^{10}$ study showed that physicians with more experience were more willing to disclose, which might imply that experienced physicians are in fact conscious and compliant with regard to hospital policies and programs.

We also found a statistically significant connection between gender and awareness of policies and programs. Male physicians were more aware of policies and

Table 4 Factors Influencing Awareness of Different Policies and Program

\begin{tabular}{|c|c|c|c|c|c|c|}
\hline \multirow[t]{3}{*}{ Variable } & \multicolumn{6}{|c|}{ Awareness } \\
\hline & \multicolumn{2}{|c|}{ “Disclosure Policy” (\%) } & \multicolumn{2}{|c|}{ “Just Culture Policy” (\%) } & \multicolumn{2}{|c|}{ “Second Victim Program" (\%) } \\
\hline & $\begin{array}{l}\text { Yes } \\
n=48\end{array}$ & $\begin{array}{l}\text { No } \\
n=156\end{array}$ & $\begin{array}{l}\text { Yes } \\
n=48\end{array}$ & $\begin{array}{l}\text { No } \\
n=156\end{array}$ & $\begin{array}{l}\text { Yes } \\
n=48\end{array}$ & $\begin{array}{l}\text { No } \\
n=156\end{array}$ \\
\hline \multicolumn{7}{|l|}{ Levels } \\
\hline Consultant & $35(57)$ & $45(32)$ & $3 I(6 \mid)$ & $49(31)$ & $30(61)$ & $50(33)$ \\
\hline Specialist & $15(24)$ & $23(16)$ & $9(19)$ & $29(19)$ & $10(20)$ & $27(18)$ \\
\hline Fellow & $0(0)$ & $7(5)$ & $0(0)$ & $7(5)$ & $0(0)$ & $7(5)$ \\
\hline Resident & $\mathrm{II}(\mathrm{I})$ & $5 \mathrm{I}(36)$ & $4(8)$ & $58(37)$ & $9(18)$ & $52(34)$ \\
\hline GP & $\mathrm{I}(2)$ & $16(11)$ & $4(8)$ & $13(8)$ & $0(0)$ & $17(\mid I)$ \\
\hline Test & \multicolumn{2}{|c|}{$x^{2}=20.803, P<0.0001$} & \multicolumn{2}{|c|}{$x^{2}=22.504, P<0.0001$} & \multicolumn{2}{|c|}{$x^{2}=18.475, P=0.001$} \\
\hline \multicolumn{7}{|l|}{ Gender } \\
\hline Female & $\mathrm{II}(\mathrm{I})$ & $49(35)$ & $9(19)$ & $5 I(33)$ & $\mathrm{II}(23)$ & $48(3 I)$ \\
\hline Male & $50(82)$ & $93(66)$ & $38(81)$ & $105(67)$ & $37(77)$ & $105(69)$ \\
\hline Test & \multicolumn{2}{|c|}{$\begin{array}{l}x^{2}=5.562, P=0.018 \\
\text { Fisher exact test, } P=0.019\end{array}$} & \multicolumn{2}{|c|}{$\begin{array}{l}x^{2}=3.182, P=0.074 \\
\text { Fisher exact test, } P=0.100\end{array}$} & \multicolumn{2}{|c|}{$\begin{array}{l}x^{2}=1.260, P=0.262 \\
\text { Fisher exact test, } P=0.283\end{array}$} \\
\hline \multicolumn{7}{|l|}{ Department } \\
\hline Medical & $32(52)$ & $75(53)$ & $26(54)$ & $81(52)$ & $26(53)$ & $8 I(53)$ \\
\hline Surgical & $30(48)$ & $67(47)$ & $22(46)$ & $75(48)$ & $23(50)$ & $72(47)$ \\
\hline Test & \multicolumn{2}{|c|}{$\begin{array}{l}x^{2}=0.025, P=0.874 \\
\text { Fisher exact test, } P=0.880\end{array}$} & \multicolumn{2}{|c|}{$\begin{array}{l}x^{2}=0.074, P=0.785 \\
\text { Fisher exact test, } P=0.869\end{array}$} & \multicolumn{2}{|c|}{$\begin{array}{l}x^{2}=0.000, P=0.988 \\
\text { Fisher exact test, } P=1.000\end{array}$} \\
\hline
\end{tabular}


programs. This finding contradicts what was found earlier by Cruickshank ${ }^{11}$ that female physicians were better at finding information, communicating and engaging patients and their families in the process of care. The gender distribution of the sample in this study might have affected this finding, as the majority of the respondents were male.

When the association between the specialty and awareness of policies and programs was tested, the results showed no statistically significant difference. The absence of a significant association could be due to a lack of appropriate sample size.

Furthermore, we explored the participants' awareness of policies and programs that support disclosure practice. The results showed that the majority of participants were aware of the "Disclosure of Unanticipated Patient's Outcome" policy, followed by the "Second Victim Program", then the "Just Culture" policy. Although the hospital started to increase awareness of disclosure in 2016, the overall awareness score was actually low. This could be due to the fact that all policies and programs were established for national and international accreditation requirements only. Thus, the health care workers did not see the actual value of disclosure in their routine jobs. In fact, the practice of disclosure as part of clinicians' tasks is new and still evolving. ${ }^{3,4}$ Moreover, not all health organizations have implemented it. Therefore, it is important to develop detailed policies including who should be involved in disclosure practice, who should do the disclosure, and when and what information should be disclosed to patients. ${ }^{6}$ Another reason behind the low awareness level could be the lack of top management support, which could influence the implementation of policies and programs. ${ }^{2}$ In fact, it might also influence the reporting of incident practice based on what was reported by Fallowfield and Fleissig. ${ }^{2}$

This study also tested the association between "Disclosure of Unanticipated Patient's Outcome" policy, "Just Culture" policy and "Second Victim Program" awareness and the actual disclosure of medical errors. There was no significant association between the awareness level of policies and the "Second Victim Program" and actual disclosure, which might be due to low awareness of policies and the "Second Victim Program." This is aligned with Gallagher's, ${ }^{6}$ study, which showed shortcomings of the full-disclosure policy actually taking place, implying that there are low awareness, knowledge and training with regard to policies related to disclosure. Despite the fact that health care providers know the importance and support of open disclosure policies, they lack knowledge about what to disclose to patients. ${ }^{3}$

Physicians at King Fahd Hospital do not disclose medical errors due to lack of awareness of the "Just Culture" policy. This was emphasized in the Institute of Medicine (IOM) report, which demonstrated that without just culture, there is no medical error disclosure and few learning opportunities. The report also stated that punitive culture can be a threat to patients, indicating the need for fundamental change in culture by adopting transparent culture, trust culture, just culture and learning culture, starting from top hospital management. ${ }^{12}$

Delbanco and Bell ${ }^{13}$ reported that health care providers suffer from guilt, fear and isolation after medical errors, and physicians usually turn away from patients who have been harmed. Sorensen et $\mathrm{al}^{3}$ also demonstrated that providers who practiced disclosure of medical errors recognized that not all physicians are comfortable and competent in doing so. This study is aligned with an Institute of Health Improvement (IHI) report showing that physicians can feel upset, guilty, self-critical and depressed after medical errors, and early identification of physicians' suffering through a second victim program can improve disclosure to patients. ${ }^{14}$ In Fallowfield and Fleissig's ${ }^{2}$ study, all physicians reported that they need help and support after medical errors, yet only one third obtained help.

Overall, the results supported the null hypothesis that policies and programs related to disclosure do not impact disclosure practice, in contrast to reports in other studies that establishing clear guidelines and policies can promote disclosure practice. ${ }^{15-18}$

It is important to highlight some limitations in conducting this research. First, this study focused only on physicians; thus, further research needs to explore the awareness of all health care providers related to disclosure practice, such as nurses, technicians, managers and risk managers. Second, in terms of sampling distribution, most of the study population was male under a specific specialty; due to time restraints, we could not test more. Lastly, the results of the study might not be generalizable because the sample was recruited only from one teaching hospital in one region. Further studies need to be conducted covering a wider sample and different hospitals.

\section{Conclusion}

Our results indicate that physicians of King Fahd Hospital of the University do not disclose medical errors to patients, 
which is linked to the low rate of awareness of policies and the "Second Victim Program." These results also resonate with the fact that disclosure policies and programs are new and require training and specific skills. To achieve an optimal level of disclosure, the hospital needs to overcome the defects and show the value of disclosure of medical errors to health care providers. Medical error disclosure can be traumatic to physicians as well as to patients, yet following disclosure policies and being supported by the "Just Culture" policy and "Second Victim Program" will ensure a less stressful process.

King Fahd Hospital leaders need to provide continuous training and education to physicians about the "Disclosure of Unanticipated Patient's Outcome" policy, "Just Culture" policy and "Second Victim Program" to increase the awareness level. They should also monitor the correct implementation of these policies. A culture of safety and transparent communication between providers, management and patients needs to be established to promote disclosure practice.

\section{Abbreviations}

US, United States; JCI, Joint Commission International; CBAHI, Central Board for Accreditation of Healthcare Institutions; KFHU, King Fahd Hospital of the University; SPSS, Statistical Package for Social Sciences; IOM, Institute of Medicine; IHI, Institute of Health Improvement.

\section{Data Sharing Statement}

The datasets during and/or analyzed during the current study available from the corresponding author on reasonable request.

\section{Ethics Approval and Consent to Participate}

Ethical approvals were obtained from the Institutional Review Board at Imam Abdulrahman bin Faisal University. Written informed consent was provided at the beginning of the survey.

\section{Acknowledgments}

We would like to thank Editage for English language editing.

\section{Author Contributions}

All authors made substantial contributions to conception and design, acquisition of data, or analysis and interpretation of data; took part in drafting the article or revising it critically for important intellectual content; gave final approval of the version to be published; and agree to be accountable for all aspects of the work.

\section{Funding}

The authors declare that there is no funding relevant to this work.

\section{Disclosure}

The authors declare that they have no competing interests.

\section{References}

1. Gallagher TH, Waterman AD, Ebers AG, Fraser VJ, Levinson W. Patients' and physicians' attitudes regarding the disclosure of medical errors. JAMA. 2003;289(8):1001-1007. doi:10.1001/jama.289.8.1001

2. Fallowfield L, Fleissig A Communication with Patients in the Context of Medical Error [Master's thesis]. University of Sussex; 2003.

3. Sorensen R, Iedema R, Piper D, Manias E, Williams A, Tuckett A. Disclosing clinical adverse events to patients: can practice inform policy? Health Expect. 2009;13:148-159. doi:10.1111/j.1369-7625. 2009.00569.x

4. Hébert PC, Levin AV, Robertson G. Bioethics for clinicians: 23. Disclosure of medical error. CMAJ. 2001;164(4):509-513.

5. King Fahd Hospital of the University. Disclosure of Unanticipated Patients' Outcome Policy at KFHU; 2016.

6. Gallagher TH, Brundage G, Bommarito KM, et al. National survey: risk managers' attitudes and experiences regarding patient safety and error disclosure. Am Soc Healthc Risk Manag. 2006;26(3):11-16. doi:10.1002/jhrm.5600260305

7. Daniel W. Biostatistics: A Foundation for Analysis in the Health Sciences. 7th ed. New York: John Wiley \& Sons; 1999.

8. SPSS Statistics for Windows. Version 19.0. IBM Corp; 2010.

9. Heale R, Twycross A. Validity and reliability in quantitative studies. Evid Based Nurs. 2015;18(3):66-67. doi:10.1136/eb-2015-102129

10. Kaldjian LC, Jones EW, Wu BJ, Forman-Hoffman VL, Levi BH, Rosenthal GE. Disclosing medical errors to patients: attitudes and practices of physicians and trainees. J Gen Intern Med. 2007;22 (7):988-996. doi:10.1007/s11606-007-0227-z

11. Cruickshank H Are women better doctors than men? Healthline; 2018. Available from: https://www.healthline.com/health-news /women-doctors-better-patient-interactions\#1. Accessed July 7, 2020.

12. Ulrich B, Kear T. Patient safety and patient safety culture: foundations of excellent health care delivery. Nephrol Nurs J. 2014;41(5):447-456.

13. Delbanco T, Bell SK. Guilty, afraid, and alone-Struggling with medical error. $N$ Engl J Med. 2007;357(17):1682-1683. doi:10.10 56/NEJMp078104

14. Institute of Health Improvement. Patient Safety 105: communicating with Patients after Adverse Event s.

15. Elwy AR, Itani KM, Bokhour BG, et al. Surgeons' disclosures of clinical adverse events. JAMA Surg. 2016;151(11):1015-1021. doi:10.1001/jamasurg.2016.1787

16. Ock M, Choi EY, Jo M, Lee S. General public's attitudes towards disclosure of patient safety incidents in Korea: results of disclosure of patient safety incidents survey I. J Patient Saf. 2017;1-6.

17. Allan A, Munro B. Open Disclosure: A Review of the Literature. Joondalup, Western Australia: Edith Cowan University; 2008:1-25.

18. Zaghloul AA, Rahman SA, El-Eneine NYA. Obligation towards medical errors disclosure at a tertiary care hospital in Dubai, UAE. Int J Risk Saf Med. 2016;28:93-99. doi:10.3233/JRS-160722 


\section{Publish your work in this journal}

Risk Management and Healthcare Policy is an international, peerreviewed, open access journal focusing on all aspects of public health, policy, and preventative measures to promote good health and improve morbidity and mortality in the population. The journal welcomes submitted papers covering original research, basic science, clinical \& epidemiological studies, reviews and evaluations, guidelines, expert opinion and commentary, case reports and extended reports. The manuscript management system is completely online and includes a very quick and fair peer-review system, which is all easy to use. Visit http://www.dovepress.com/testimonials.php to read real quotes from published authors.

Submit your manuscript here: https://www.dovepress.com/risk-management-and-healthcare-policy-journal 